\title{
Potential Application of Measuring Serum Infliximab Levels in Rheumatoid Arthritis Management: A Retrospective Study based on KURAMA Cohort Data
}

Kazuto Nakae

Kyoto University

Sho Masui

Kyoto University

Atsushi Yonezawa ( $\nabla$ ayone@kuhp.kyoto-u.ac.jp )

Kyoto University

Motomu Hashimoto

Kyoto University

Ryu Watanabe

Kyoto University

Koichi Murata

Kyoto University

Kosaku Murakami

Kyoto University

Masao Tanaka

Kyoto University

Hiromu Ito

Kyoto University

Kotoko Yokoyama

Shimadzu Corporation (Japan)

Noriko Iwamoto

Shimadzu Corporation (Japan)

Takashi Shimada

Shimadzu Corporation (Japan)

Miyuki Nakamura

Kyoto University

Masaya Denda

Kyoto University

Kotaro Itohara

Kyoto University

Shunsaku Nakagawa 
Kyoto University

\section{Yasuaki Ikemi}

Kyoto University

\section{Satoshi Imai}

Kyoto University

\section{Takayuki Nakagawa}

Kyoto University

\section{Makoto Hayakari}

Kyoto University

\section{Kazuo Matsubara}

Kyoto University

\section{Research Article}

Keywords: Rheumatoid arthritis, Infliximab, Therapeutic Drug Monitoring, Cohort study

Posted Date: April 8th, 2021

DOl: https://doi.org/10.21203/rs.3.rs-396661/v1

License: (c) (7) This work is licensed under a Creative Commons Attribution 4.0 International License. Read Full License 
1 Potential application of measuring serum infliximab levels in rheumatoid arthritis

4 Kazuto Nakae ${ }^{1,2 \#}$, Sho Masui ${ }^{1,2 \#}$, Atsushi Yonezawa ${ }^{1,2^{*}}$, Motomu Hashimoto ${ }^{3}$, Ryu

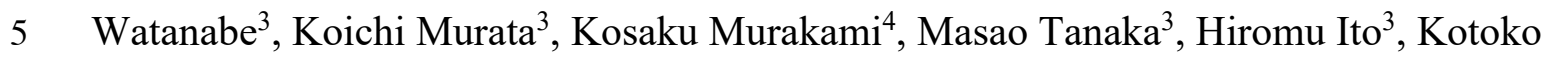

6 Yokoyama ${ }^{5}$, Noriko Iwamoto ${ }^{5,6}$, Takashi Shimada ${ }^{5,6}$, Miyuki Nakamura ${ }^{1,2}$, Masaya

7 Denda $^{1,2}$, Kotaro Itohara ${ }^{1}$, Shunsaku Nakagawa ${ }^{1}$, Yasuaki Ikemi ${ }^{1}$, Satoshi Imai ${ }^{1}$,

8 Takayuki Nakagawa ${ }^{1}$, Makoto Hayakari $^{1}$, Kazuo Matsubara $^{1}$

9

10 Author Affiliations:

11 1. Department of Clinical Pharmacology and Therapeutics, Kyoto University

12 Hospital, Japan

13 2. Graduate School of Pharmaceutical Sciences, Kyoto University, Japan

14 3. Department of Advanced Medicine for Rheumatic Diseases, Graduate School of

15 Medicine, Kyoto University, Japan

16 4. Department of Rheumatology and Clinical Immunology, Graduate School of

17 Medicine, Kyoto University, Japan.

18 5. Shimadzu Corporation, Kyoto, Japan

19 6. Shimadzu Scientific Instruments, Bothell, Washington, USA 
*Corresponding author: Atsushi Yonezawa, Ph.D.

606-8507, Japan 


\section{Abstract}

\section{Background}

32 Infliximab (IFX) therapy has considerably improved the treatment of rheumatoid 33 arthritis (RA). On the other hand, in some patients, the efficacy of IFX therapy is not 34 adequate, or gradually diminishes with the lapse of the treatment. Although previous 35 studies have reported a positive relationship between serum IFX levels and therapeutic 36 efficacy, the potential application of IFX therapeutic drug monitoring (TDM) in clinical 37 practice remains unclear. The purpose of this study was to investigate the potential applications of IFX TDM by analyzing a Japanese cohort database.

\section{Methods}

40 Data were collected retrospectively from the Kyoto University Rheumatoid Arthritis

41 Management Alliance, KURAMA, cohort between January 1, 2011, and December 31, 42 2018. Serum IFX levels were measured using liquid chromatography-tandem mass 43 spectrometry.

\section{Results}

45 Out of the 311 RA patients who received IFX therapy, 41 were eligible for analysis.

46 Serum IFX levels were significantly higher in responders than in non-responders. An

47 optimal cut-off value was determined to be $0.4 \mu \mathrm{g} / \mathrm{mL}$ based on a receiver operating 48 characteristic curve. At the IFX measurement point, a better therapeutic response was 
49 observed in the High-IFX group $(n=31)$ than in the Low-IFX group $(n=10)$. Conversely,

50 at the maximum effect point, when DAS28-ESR (the 28 joint disease activity score

51 incorporating erythrocyte sedimentation rate) was the lowest between IFX introduction

52 and measurement points, there were no differences in responder proportions between the

53 Low- and High-IFX groups.

\section{Conclusions}

55 In clinical practice, IFX primary ineffectiveness could be avoided with appropriate dose

56 escalation without blood concentration measurement. However, IFX TDM could

57 facilitate the identification of secondary non-responders, and in turn, proper IFX use.

59 Keywords: Rheumatoid arthritis; Infliximab; Therapeutic Drug Monitoring; Cohort

60 study

61 


\section{Background}

63 Infliximab (IFX) is a chimeric monoclonal antibody composed of human constant and

64 murine variable regions that specifically bind to tumor necrosis factor alpha (TNF- $\alpha$ ).

65 IFX therapy has substantially improved the treatment of rheumatoid arthritis (RA). The

66 result of Anti-Tumor Necrosis Factor Trial in Rheumatoid Arthritis with Concomitant

67 Therapy (ATTRACT) study has revealed that IFX therapy provided clinical benefits and

68 halted joint damage progression $[1,2]$. However, in some patients, the efficacy of IFX

69 therapy is not adequate, or is gradually lost with the lapse of the treatment [3-6]. It has

70 also been reported that secondary non-response occurs in approximately a half of RA

71 patients during the first year of its treatment [7]. In addition, another study has shown that

72 IFX discontinuation rate due to inefficacy was $32.1 \%$ at 36 months [8]. The current

73 challenge in IFX therapy is the optimization of long-term treatment.

74 The pharmacokinetic mechanisms of therapeutic antibodies have largely been clarified.

75 The development of anti-drug antibodies (ADAs) is associated with low serum drug

76 levels and non-response [9-11]. Previous studies have shown that approximately $10-60 \%$

77 of RA patients receiving IFX developed ADAs against IFX within the first 6 months [12-

78 15]. In addition to ADAs, baseline TNF- $\alpha$ level is another factor that reduces serum IFX

79 levels [16]. Furthermore, FcRn (neonatal Fc receptor) function influences the

80 pharmacokinetics of therapeutic antibodies [17, 18]. High inter- and intra-individual 
81 variabilities in monoclonal antibody pharmacokinetics have been reported [19].

82 Consequently, therapeutic strategies that take into account IFX pharmacokinetics

83 variability should be developed.

84 Therapeutic drug monitoring (TDM) has facilitated the optimal and appropriate use of

85 immunosuppressive drugs and antiepileptic drugs, etc. Based on the serum concentrations

86 of drugs, dosages can be adjusted to appropriate therapeutic concentrations and ranges.

87 Some studies have demonstrated that clinical responses to IFX therapy are associated

88 with serum IFX levels. A prospective, randomized, double-blind study (the RISING

89 study) has reported a significant correlation between serum IFX levels and disease

90 activity score in 28 joints (DAS28)-remission [20]. A non-interventional retrospective

91 study has also reported that high serum IFX levels are related to good responses at 52

92 weeks from baseline [15]. Although a relationship between serum IFX levels and its

93 therapeutic benefits has been described in several studies [15, 20-22], it remains unclear

94 how IFX TDM could be applied in clinical practice.

95 Here, we conducted a retrospective cohort study by enrolling consecutive RA patients

96 treated with IFX in a cohort, and investigated the practicality of IFX TDM in clinical

97 practice. Furthermore, we measured ADA levels to evaluate its correlation with serum

98 IFX levels.

99 


\section{Patients}

102 The study subjects were enrolled from the Kyoto University Rheumatoid Arthritis Management Alliance (KURAMA) cohort, which was established in 2011 by the Center for Rheumatic Diseases at Kyoto University Hospital. The cohort aims to provide strict RA control and to use patient clinical and laboratory data in clinical investigations, as described previously [23]. All patients fulfilled the revised 1987 American College of Rheumatology (ACR) or the 2010 ACR/European League Against Rheumatism (EULAR) classification criteria for RA. Informed consent to enroll in this retrospective cohort study was obtained from all the patients. All data were de-identified and analyzed anonymously. The present study adhered to the principles of the Declaration of Helsinki,

111 and was approved by the Medical Ethics Committee of Kyoto University Graduate School and Faculty of Medicine (R0357).

113 KURAMA cohort data between January 1, 2011 and December 31, 2018 were used in

114 the present study. Out of the 311 RA patients with IFX therapy, 210 were excluded, 115 because their serum IFX levels were not obtained during maintenance therapy (at least 116112 days after IFX introduction). In addition, 55 patients were excluded due to lack of the 117 28-joint disease activity score incorporating erythrocyte sedimentation rate (DAS28118 ESR) data 56 days before IFX introduction and 56 days before or after IFX measurement. 
119 Five patients who had already completed clinical remission (DAS28-ESR < 2.6) before

120 IFX introduction were also excluded, and 41 patients were eligible for further analysis

121 (Figure 1).

122

123

\section{Data collection and evaluation of disease activity}

124 Clinical characteristics included age, body weight, sex, RA disease duration, IFX

125

126

128

129

131

132 treatment duration, weekly methotrexate (MTX) dose, oral glucocorticoid use, conventional synthetic disease modifying anti-rheumatic-drug (csDMARD) use, tender joint count, swollen joint count, C-reactive protein (CRP) level, and rheumatoid factor (RF). Actarit, aurothiomalate, auranofin, bucillamine, iguratimod, leflunomide, mizoribine, salazosulfapyridin, cyclosporine, and tacrolimus were considered as csDMARDs. RA disease activity was evaluated based on clinical disease activity index (CDAI), simplified disease activity index (SDAI), physical disability by health assessment questionnaire-disability index (HAQ-DI), and DAS28-ESR. Baseline was defined as the last data within 3 months before IFX introduction. Patients achieving good or moderate responses to IFX therapy according to the EULAR response criteria were defined as "responders," and patients with no response were defined as "non-responders." 

before a new infusion. Serum IFX levels were measured using an LCMS-8060 quadrupole modifications [24-26]. Briefly, to obtain the peptides from the fragment antigen-binding

142 (Fab) region of immunoglobulin $\mathrm{G}$, serum samples were pretreated using the $\mathrm{nSMOL}^{\mathrm{TM}}$ 143 Antibody BA Kit (SHIMADZU, Kyoto, Japan) according to the provided protocol. The 144 lower limit of quantitation was $0.293 \mu \mathrm{g} / \mathrm{mL}$.

\section{Detection of ADAs in serum}

147 ADA analysis was performed by the electrochemiluminescence (ECL) method [27, 28].

148 A microplate coated with streptavidin (MSD GOLD 96-well Streptavidin QUICKPLEX

149 Plate, Meso Scale Diagnostics [MSD], Rockville, MD, USA) was blocked with $150 \mu \mathrm{L}$ blocking solution ( $3 \%$ MSD Blocker A) overnight at $4{ }^{\circ} \mathrm{C}$. A master mixture of $20 \mu \mathrm{g} / \mathrm{mL}$ biotinylated IFX and $20 \mu \mathrm{g} / \mathrm{mL}$ ruthenium-labeled IFX was prepared in assay diluent ( $1 \%$ MSD Blocker A) at a ratio of 1:1. Subsequently, $25 \mu \mathrm{L}$ of a diluted sample and $50 \mu \mathrm{L}$ of 153 the master mixture were added to each well in a 96-well plate, and incubated for $2 \mathrm{~h}$ under gentle agitation. After three washes with $200 \mu \mathrm{L}$ of wash buffer (phosphate-buffered saline with $0.05 \%$ Tween 20 ), $50 \mu \mathrm{L}$ of premix solution was transferred to each corresponding streptavidin-coated plate well, and the plates were incubated for $1 \mathrm{~h}$ under 
agitation. The plates were then washed three times, and $150 \mu \mathrm{L}$ of read buffer (MSD Read

158 Buffer T [4× ] diluted two-fold in ultrapure water) was added to each well. The ECL signal

159 from the solution was measured using a MESO QuickPlex SQ120 (MSD).

\section{Statistical analysis}

162 Statistical analyses were performed using GraphPad Prism v7.0 (GraphPad Software,

163 Inc., La Jolla, CA, USA). Non-normally distributed data were summarized with medians

164 and analyzed using nonparametric tests (Mann-Whitney U test or Wilcoxon signed-rank

test). Categorical data summarized with percentages were analyzed using Fisher's exact

test with continuity correction, where necessary. Results were considered statistically

significant at $p$-value $\leq 0.05$. The Kaplan-Meier method was performed to evaluate time

to first response and time to loss of response.

169 To define an optimal cut-off value for predicting clinical response, a receiver operating USA).

\section{Results}

\section{Clinical efficacy and serum levels of IFX in RA patients}

Figure 2a shows a change in DAS28-ESR after introduction of IFX. Large inter- and 
176 intra-individual differences in daily disease activities were observed. Kaplan-Meier

177 curves indicated that more than $80 \%$ of total patients responded within 12 weeks after

178 IFX introduction in clinical practice (Figure $2 b$ ), and around $40 \%$ of responders exhibited

179 loss of response within 48 weeks after the first response (Figure 2c).

180 There were 34 responders and 7 non-responders at the measurement point (Figure 3a).

181 Serum IFX levels were significantly higher in responders than in non-responders (Figure

$1823 \mathrm{~b}$ ). The area under the curve (AUC) of the ROC curve was 0.87 , and the cut-off value

183 that distinguished EULAR responders from non-responders was $0.319 \mu \mathrm{g} / \mathrm{mL}$

184 (sensitivity: $94.1 \%$, specificity: $85.7 \%$, Figure $3 \mathrm{c}$ ). Considering clinical usefulness, 0.4

$185 \mu \mathrm{g} / \mathrm{mL}$ was selected as the cut-off value in subsequent analyses.

186

187 Background demographics and clinical characteristics of patients

188 Patients were divided into two groups based on serum IFX levels, that is, patients with serum IFX level $<0.4 \mu \mathrm{g} / \mathrm{mL}$ (Low-IFX group, $\mathrm{n}=10$ ) and patients with serum IFX level

$190 \geq 0.4 \mu \mathrm{g} / \mathrm{mL}$ (High-IFX group, $\mathrm{n}=31$ ). The baseline demographics and clinical 191 characteristics of the patients in the two groups are summarized in Table 1. At the measurement point, the mean duration of IFX treatment was around 1 year. Age, CDAI, and SDAI were significantly lower in the Low-IFX group than in the High-IFX group, and only patients in the High-IFX group used oral glucocorticoids. There were no 
significant differences in body weight, sex, disease duration, duration of treatment, tender joint count, swollen joint count, CRP level, number of antibodies to citrullinated peptide antigens-positive or RF-positive patients, HAQ-DI, DAS28-ESR, and concomitant MTX and csDMARD use.

\section{Disease activity markers in Low-IFX group and High-IFX group}

In the present study, the "maximum effect point" was defined as the date when DAS28-

ESR was the lowest during the IFX therapy between after its introduction point and at the measurement point. At the maximum effect point, only two patients $(4.9 \%)$ were nonresponders; there were no differences in proportions of responders between the Low-IFX and High-IFX groups ( $p=0.43$, Table 2). However, at the measurement point, five patients additionally turned into non-responder status, accordingly, there were significant differences in responder proportions between the Low-IFX group and the High-IFX group based on Fisher's exact test $(p<0.01)$. One non-responder in the High-IFX group had finally attained efficacy after the measurement point. Disease activity marker trends between the introduction and measurement points in the two groups are illustrated in Figure 4. CDAI and SDAI in the Low-IFX group improved significantly. In addition, CDAI, SDAI, CRP, and HAQ-DI scores in the High-IFX group exhibited notable improvements. 
215 Correlation between serum IFX levels and ADA positivity

216 In 39 of the 41 investigated patients, serum samples were sufficient amount for the

217 ADA determination. ADA was detected in four patients $(10.3 \%)$ at the measurement

218 point. The IFX levels in the ADA-positive group were significantly lower than that in

219 the ADA-negative group ( $p<0.01$, Figure 5). Although two patients in the ADA-

220 positive group (50.0\%) were responders, 30 patients in the ADA-negative group

$221(85.7 \%)$ were responders. There were no significant differences in proportions of responders between the ADA-positive group and ADA-negative group (Table 2).

\section{Discussion}

225 In a previous intervention study (the RISING study), RA patients were randomly assigned to three treatment groups (3, 6, and $10 \mathrm{mg} / \mathrm{kg}$ IFX infusions) at week 10 after receiving $3 \mathrm{mg} / \mathrm{kg}$ IFX at weeks 0,2 , and 6 [20]. The rates of responders at week 54 for

3, 6 and $10 \mathrm{mg} / \mathrm{kg}$ were $10 \%, 56 \%$ and $100 \%$, respectively. Better response was obtained

229 in patients with higher dose of IFX. In addition, when the serum IFX concentration was

$230 \geq 1.0 \mu \mathrm{g} / \mathrm{mL}$, a clinical response was observed in $98.8 \%$ of patients. Although the exact therapeutic window of IFX is yet to be clearly defined, a higher trough level has been associated with improved clinical outcomes in several observational studies and post-hoc 
analyses of clinical trials across different diseases [21, 22, 29-32]. Notably, our real-world

234 cohort data indicated the effectiveness of IFX treatment in 39 of the 41 target patients

$235(95.1 \%)$ at the point of maximum effect. The results obtained from this study strongly

236 suggested that physicians successfully increased IFX doses to appropriate levels in each

237 patient even without measuring blood levels, and that primary ineffectiveness could be

238 avoided in clinical practice.

239 Conversely, some patients showed secondary loss of response to IFX with the lapse of

240 the continuous use. Notably, at the measurement point, the efficacy was significantly

241 lower in the Low-IFX group than in the High-IFX group, strongly suggesting that that the

242 effect of this therapy was potentially decreased by lower blood IFX level. In clinical

243 practice, checking DAS28-ESR is somewhat difficult for physicians due to requiring

244 considerable time for examination, especially on busy patients. Large inter- and intra-

245 individual differences in disease activities were observed. Consequently, it is challenging

246 to reliably determine secondary ineffectiveness under long-term use. Overall, we propose

247 the development of a treatment algorithm based on IFX TDM, wherein IFX therapeutic

248 efficacy would be extensively re-evaluated when blood IFX concentrations are low under

249 continuous use.

250 The determination of a cut-off value for predicting clinical response is a key challenge.

251 In the RISING study, a trough serum IFX level of $1.0 \mu \mathrm{g} / \mathrm{mL}$ was the threshold level for 
252 eliciting clinical responses [20]. Wolbink et al. [33] reported similar results, where 253 patients with low trough serum IFX levels (less than $1.2 \mu \mathrm{g} / \mathrm{mL}$ ) showed relatively low 254 improvements in DAS28 score. From the result of ROC analysis in this study, an optimal 255 cut-off value of $\geq 0.4 \mu \mathrm{g} / \mathrm{mL}$ was determined. Our study also revealed almost similar 256 results when the cut-off value was determined to be at serum IFX level $\geq 1.0 \mu \mathrm{g} / \mathrm{mL}$ 257 (Additional file 1 Table S1), which is largely consistent with RISING study [20]. 258 Enzyme-linked immunosorbent assay method has been extensively used to quantify 259 serum therapeutic antibodies. However, by use of this technique, nonspecific signals could be detected $[34,35]$. In the present study, we employed a liquid chromatographytandem mass spectrometry (LC-MS/MS) method with nano-surface and molecularorientation limited proteolysis to monitor IFX-specific peptides, based on Food and Drug 263 Administration (FDA) criteria [36]. The analytical methods used should be taken into 264 account to set cut-off values in clinical practice. Further studies are required to determine the optimal cut-off values across several analytical methods.

266 Previous studies have shown that ADA is one of the factors influencing IFX 267 pharmacokinetics [9-11]. ADA formation increases IFX clearance, which can, in turn, reduce serum IFX levels. In the present study, 4 out of 39 patients $(10.3 \%)$ were ADA269 positive. Compared to the ADA-negative patients, the ADA-positive patients had 270 significantly lower serum IFX levels. The proportion of patients satisfying the EULAR 
response criteria tended to be lower in the ADA-positive group. Although ADA could influence IFX pharmacokinetics, the key factor influencing IFX efficacy is serum IFX level. Although there are factors other than ADA to influence blood IFX levels, 274 monitoring IFX levels is the potentially optimal tool for evaluating its clinical efficacy.

275 Conversely, dose escalation of IFX could be less successful for improving treatment 276 efficacy in ADA-positive patients compared to that in ADA-negative patients [37, 38]. Measurement of ADA as well as serum IFX concentrations, could facilitate determination 278 of the next appropriate therapeutic strategy between dose escalation or switching therapies in patients exhibiting secondary loss of response.

280 The present study had some limitations. First, the sample size was small. We had to exclude numerous patients with no information on serum IFX level or DAS28-ESR data around the IFX administration date. Second, we did not measure serum IFX levels at the maximum effect point and were unable to investigate the association between reduction in DAS28-ESR and serum IFX levels at the maximum effect point. Third, background characteristics in several patient were different between the High-IFX and Low-IFX randomization to control or eliminate confounding factors. However, more than $90 \%$ of patients in both the High- and Low-IFX groups exhibited a primary response. The skewed 
patient characteristic distributions could have had relatively less impact on our results associated with secondary non-response.

\section{Conclusions}

In the present study, we demonstrated that serum IFX levels were correlated with IFX therapeutic efficacy under continuous use, based on real-world cohort data. In clinical practice, the IFX primary ineffectiveness could be avoided via appropriate dose escalation without measuring the blood concentrations. However, IFX TDM could facilitate the identification of secondary non-response and, in turn, proper IFX use.

\section{List of abbreviations}

ACR: American College of Rheumatology; ADA: Anti-drug antibody; CDAI: Clinical disease activity index; CRP: C-reactive protein; csDMARD: Conventional synthetic disease modifying anti-rheumatic drug; DAS28-ESR: The 28 joint disease activity score incorporating erythrocyte sedimentation rate; ECL: Electrochemiluminescence; EULAR:

European League Against Rheumatism; FcRn; Neonatal Fc receptor; FDA: Food and Drug Administration; HAQ-DI: Health assessment questionnaire-disability index; IFX: Infliximab; LC-MS/MS, liquid chromatography-tandem mass spectrometry; MTX: Methotrexate ; RA: Rheumatoid arthritis; RF: Rheumatoid factor; ROC: Receiver 
operating characteristic; SD: Standard deviation; SDAI: Simplified disease activity index;

309 TDM: Therapeutic drug monitoring; TNF- $\alpha$ : Tumor necrosis factor alpha

\section{Declarations}

312 Ethics approval and consent to participate: The present study adhered to the principles

313 of the Declaration of Helsinki, and was approved by the Medical Ethics Committee of

314 Kyoto University Graduate School and Faculty of Medicine (R0357). Informed consent

315 to enroll in the retrospective cohort study was obtained from all the patients. All data were

316 de-identified and analyzed anonymously.

318 Consent for publication: Not applicable.

Availability of data and materials: The datasets used and/or analyzed during the current study are available from the corresponding author on reasonable request.

323 Competing interests: M.H., R.W., K.Murata, M.T., and H.I. are associated with a department financially supported by two local governments in Japan (Nagahama City,

325 Shiga and Toyooka City, Hyogo) and five pharmaceutical companies (Mitsubishi

326 Tanabe Pharma Corp., Chugai Pharmaceutical Co., Ltd., Ayumi Pharmaceutical Corp., 
327 Asahi Kasei Pharma Corp., and UCB Japan Co., Ltd.). A.Y. and K.Matsubara has

328 received a research grant from Shimadzu Corporation. M.H. received a research grant

329 and/or speaker fee from Bristol-Myers Squibb Co., Eisai Co., Ltd., Eli Lilly Japan K.K.,

330 and Mitsubishi Tanabe Pharma Corp. R.W. has received speaker's fee from Mitsubishi

331 Tanabe Pharma Corp., Pfizer Inc., Sanofi K.K., AbbVie GK, Asahi-Kasei Corp., Eisai

332 Co., Ltd., Eli Lilly Japan K.K., Bristol-Myers Squibb Co., and Janssen Pharmaceutical

333 K.K. K.Murata received a speaking fee from Eisai Co., Ltd., Mochida Pharmaceutical

334 Co., Ltd., and Astellas Pharma Inc. M.T. has received research grants and/or speaker

335 fees from AbbVie GK, Asahi Kasei Pharma Corp., Astellas Pharma Inc., Ayumi

336 Pharmaceutical Corp., Chugai Pharmaceutical Co., Ltd., Eisai Co., Ltd., Eli Lilly Japan

337 K.K., Janssen Pharmaceutical K.K., Mitsubishi Tanabe Pharma Corp., Novartis Pharma

338 K.K., Pfizer Inc., Taisho Pharma Co., Ltd., and UCB Japan Co., Ltd. H.I. has received a

339 research grant and/or speaker fees from Bristol-Myers Squibb Co., Eisai Co., Ltd.,

340 Taisho Pharmaceutical Co., Ltd., Mochida Pharmaceutical Co., Ltd., and Asahi Kasei

341 Corp. The other authors declare no conflicts of interest.

342

343 Funding: This study was supported in part by a Grant-in-Aid for Scientific Research

344 (KAKENHI) from the Ministry of Education, Culture, Sports, Science and Technology

345 of Japan (Grants-in-Aid for Scientific Research (B) [19H03389]), by Japan Agency for 

or analysis of the data, the writing of the manuscript or decision to submit the manuscript

350 for publication.

Authors' contributions: A.Y., M.H., and K.Matsubara. conceived and designed the research. K.N., S.M., A.Y., M.H., and K.Matsubara. mainly contributed to writing the manuscript. M.H., R.W., K.Murata., K.Mmurakami., M.T., and H.I. recruited the patients and obtained samples. K.Y., N.I., and T.S. measured the plasma concentrations of IFX.

K.N., S.M., A.Y., M.N., M.D, K.I., S.N., Y.I., S.I., T.N., and M.H. carried out and analyzed all experiments. All the authors participated in the discussion of the results, and all had reviewed the manuscript.

Acknowledgments: The authors are grateful to Dr. Akiko Ishii-Watabe, Dr. Hiroko

Shibata, and Ms. Kazuko Nishimura, National Institute of Health Sciences, for advice on 


\section{References}

366 1. Maini R, St Clair EW, Breedveld F, Furst D, Kalden J, Weisman M, et al. Infliximab 367 (chimeric anti-tumour necrosis factor alpha monoclonal antibody) versus placebo in

6. Yanai H, Hanauer SB. Assessing response and loss of response to biological 
therapies in IBD. Am J Gastroenterol. 2011;106(4):685-98.

385 7. Buch MH, Bingham SJ, Bryer D, Emery P. Long-term infliximab treatment in rheumatoid arthritis: subsequent outcome of initial responders. Rheumatology (Oxford). 2007;46(7):1153-6.

8. Ebina K, Hashimoto M, Yamamoto W, Ohnishi A, Kabata D, Hirano T, et al. Drug retention and discontinuation reasons between seven biologics in patients with rheumatoid arthritis -The ANSWER cohort study. PLoS One. 2018;13(3):e0194130.

9. Baert F, Noman M, Vermeire S, Van Assche G, D' Haens G, Carbonez A, et al. Influence of immunogenicity on the long-term efficacy of infliximab in Crohn's disease. N Eng1 J Med. 2003;348(7):601-8.

10. Plasencia C, Jurado T, Villalba A, Peitedado D, Casla MT, Nuno L, et al. Effect of infliximab dose increase in rheumatoid arthritis at different trough concentrations: A cohort study in clinical practice conditions. Front Med. 2015;2:71.

11. Kalden JR, Schulze-Koops H. Immunogenicity and loss of response to TNF inhibitors: implications for rheumatoid arthritis treatment. Nat Rev Rheumatol. 2017;13(12):707-18.

12. Bendtzen K, Geborek P, Svenson M, Larsson L, Kapetanovic MC, Saxne T. Individualized monitoring of drug bioavailability and immunogenicity in rheumatoid arthritis patients treated with the tumor necrosis factor alpha inhibitor 
infliximab. Arthritis Rheum. 2006;54(12):3782-9.

404

405

406

407

408

409

410

411

412

413

414

415

416

417

13. Pascual-Salcedo D, Plasencia C, Ramiro S, Nuno L, Bonilla G, Nagore D, et al. Influence of immunogenicity on the efficacy of long-term treatment with infliximab in rheumatoid arthritis. Rheumatology (Oxford). 2011;50(8):1445-52.

14. Strand V, Balsa A, Al-Saleh J, Barile-Fabris L, Horiuchi T, Takeuchi T, et al. Immunogenicity of biologics in chronic inflammatory diseases: a systematic review. BioDrugs. 2017;31(4):299-316.

15. Siljehult F, Arlestig L, Eriksson C, Rantapaa-Dahlqvist S. Concentrations of infliximab and anti-drug antibodies in relation to clinical response in patients with rheumatoid arthritis. Scand J Rheumatol. 2018;47(5):345-50.

16. Takeuchi T, Miyasaka N, Tatsuki Y, Yano T, Yoshinari T, Abe T, et al. Baseline tumour necrosis factor alpha levels predict the necessity for dose escalation of infliximab therapy in patients with rheumatoid arthritis. Ann Rheum Dis. 2011;70(7):1208-15.

17. Tzaban S, Massol RH, Yen E, Hamman W, Frank SR, Lapierre LA, et al. The recycling and transcytotic pathways for IgG transport by FcRn are distinct and display an inherent polarity. J Cell Bio. 2009;185(4):673-84.

18. Kuo TT, Aveson VG. Neonatal Fc receptor and IgG-based therapeutics. MAbs. 2011;3(5):422-30. 
19. Gill KL, Machavaram KK, Rose RH, Chetty M. Potential sources of inter-subject variability in monoclonal antibody pharmacokinetics. Clin Pharmacokinet. 2016;55(7):789-805.

20. Takeuchi T, Miyasaka N, Inoue K, Abe T, Koike T. Impact of trough serum level on radiographic and clinical response to infliximab plus methotrexate in patients with rheumatoid arthritis: results from the RISING study. Mod Rheumatol. 2009;19(5):478-87.

21. Mulleman D, Meric JC, Paintaud G, Ducourau E, Magdelaine-Beuzelin C, Valat JP, et al. Infliximab concentration monitoring improves the control of disease activity in rheumatoid arthritis. Arthritis Res Ther. 2009;11(6):R178.

22. Price S. Rheumatoid arthritis: Monitoring serum concentration of infliximab might improve ra disease control. Nat Rev Rheumatol. 2010;6(2):66.

23. Terao C, Hashimoto M, Yamamoto K, Murakami K, Ohmura K, Nakashima R, et al. Three groups in the 28 joints for rheumatoid arthritis synovitis--analysis using more than 17,000 assessments in the KURAMA database. PLoS One. 2013;8(3):e59341.

24. Iwamoto N, Shimada T, Umino Y, Aoki C, Aoki Y, Sato TA, et al. Selective detection of complementarity-determining regions of monoclonal antibody by limiting protease access to the substrate: nano-surface and molecular-orientation limited proteolysis. Analyst. 2014;139(3):576-80. 
25. Iwamoto N, Yokoyama K, Takanashi M, Yonezawa A, Matsubara K, Shimada T. Verification between original and biosimilar therapeutic antibody infliximab using nSMOL coupled LC-MS bioanalysis in human serum. Curr Pharm Biotechnol. 2018;19(6):495-505.

26. Iwamoto N, Takanashi M, Yokoyama K, Yonezawa A, Denda M, Hashimoto M, et al. Multiplexed monitoring of therapeutic antibodies for inflammatory diseases using Fab-selective proteolysis nSMOL coupled with LC-MS. J Immunol Methods. 2019;472:44-54.

27. Reinisch W, Jahnsen J, Schreiber S, Danese S, Panés J, Balsa A, et al. Evaluation of the cross-reactivity of antidrug antibodies to CT-P13 and infliximab reference product (Remicade): an analysis using immunoassays tagged with both agents. BioDrugs. 2017;31(3):223-37.

28. Shibata H, Nishimura K, Miyama C, Tada M, Suzuki T, Saito Y, et al. Comparison of different immunoassay methods to detect human anti-drug antibody using the WHO erythropoietin antibody reference panel for analytes. J Immunol Methods. $2018 ; 452: 73-7$.

29. Pallagi-Kunstar E, Farkas K, Szepes Z, Nagy F, Szucs M, Kui R, et al. Utility of serum TNF- $\alpha$, infliximab trough level, and antibody titers in inflammatory bowel disease. World J Gastroenterol. 2014;20(17):5031-5. 
30. Dannepond C, Maruani A, Machet L, Ternant D, Paintaud G, Samimi M. Serum infliximab concentrations in psoriatic patients treated with infliximab: a systematic review. Acta Derm Venereol. 2015;95(4):401-6.

31. Vande Casteele NV, Ferrante M, Van Assche G, Ballet V, Compernolle G, Van Steen $\mathrm{K}$, et al. Trough concentrations of infliximab guide dosing for patients with inflammatory bowel disease. Gastroenterology. 2015;148(7):1320-9.e3.

32. Warman A, Straathof JW, Derijks LJ. Therapeutic drug monitoring of infliximab in inflammatory bowel disease patients in a teaching hospital setting: results of a prospective cohort study. Eur J Gastroenterol Hepatol. 2015;27(3):242-8.

33. Wolbink GJ, Voskuyl AE, Lems WF, De Groot E, Nurmohamed MT, Tak PP, et al. Relationship between serum trough infliximab levels, pretreatment $\mathrm{C}$ reactive protein levels, and clinical response to infliximab treatment in patients with rheumatoid arthritis. Ann Rheum Dis. 2005;64(5):704-7.

34. Güven E, Duus K, Lydolph M, Jørgensen CS, Laursen I, Houen G. Non-specific binding in solid phase immunoassays for autoantibodies correlates with inflammation markers. J Immunol Methods. 2014;403(1-2):26-36.

35. Jourdil JF, Lebert D, Gautier-Veyret E, Lemaitre F, Bonaz B, Picard G, et al. Infliximab quantitation in human plasma by liquid chromatography-tandem mass spectrometry: Towards a standardization of the methods? Anal Bioanal Chem. 
36. U.S. Food and Drug Administration. Bioanalytical Method Validation Guidance for Industry. 2018. https://www.fda.gov/regulatory-information/search-fda-guidancedocuments/bioanalytical-method-validation-guidance-industry. Accessed 5 Jan 2021.

37. Kothari MM, Nguyen DL, Parekh NK. Strategies for overcoming anti-tumor necrosis factor drug antibodies in inflammatory bowel disease: Case series and review of literature. World J Gastrointest Pharmacol Ther. 2017;8(3):155-61.

38. Afif W, Loftus Jr EV, Faubion WA, Kane SV, Bruining DH, Hanson KA, et al. Clinical utility of measuring infliximab and human anti-chimeric antibody concentrations in patients with inflammatory bowel disease. Am J Gastroenterol.

\section{Figure legends}

494 Fig. 1 Flowchart on patient inclusion and exclusion.

495 Abbreviations: IFX, infliximab; DAS28-ESR, the 28 joint disease activity score 496 incorporating erythrocyte sedimentation rate; RA, rheumatoid arthritis. 
498 Fig. 2 Clinical efficacy of IFX. (a) Change in DAS28-ESR over time. The x-axis 499 represents time after introduction of IFX. The y-axis represents values of DAS28-ESR.

500 (b) Kaplan-Meier curve showing time to first response. Responders represent patients 501 with "good or moderate response" based on the EULAR response criteria. The baseline 502 (Day 0) was defined as the day of IFX introduction. (c) Kaplan-Meier curve showing time 503 to loss of response. A change from responder to non-responder was defined as "loss of 504 response." The baseline (Day 0) was defined as the time point when the first response 505 was observed. Patients who never showed any response during observation periods were 506 excluded $(\mathrm{n}=40)$.

507 Abbreviations: DAS28-ESR, the 28 joint disease activity score incorporating erythrocyte sedimentation rate; EULAR, European League Against Rheumatism; IFX, infliximab.

510 Fig. 3 DAS28-ESR values and serum IFX concentrations in responders and non511 responders. (a) DAS28-ESR values at baseline and at IFX measurement point. 512 Responders (open circle) represent patients with "good or moderate response" and non513 responders (closed circle) represent those with "no response" based on the European 514 League Against Rheumatism (EULAR) response criteria. (b) Serum IFX levels were 515 measured in non-responders and responders. Each dot represents each patient's serum 516 IFX level and the bars indicate the median. Differences between the groups were assessed 
517 by Mann-Whitney U test. (c) Receiver operating characteristic (ROC) curve for the 518 determination of the optimal IFX cut-off value for predicting persistent responder (area 519 under the curve $(\mathrm{AUC})=0.87)$.

520 Abbreviations: DAS28-ESR, the 28 joint disease activity score incorporating erythrocyte 521 sedimentation rate; IFX, infliximab.

Fig. 4 Changes in (a) CDAI, (b) SDAI, (c) CRP, and (d) HAQ-DI from the baseline to the 524 IFX measurement point. The left figures show the data of patients with IFX level $<0.4$ $\mu \mathrm{g} / \mathrm{mL}$ (closed circles), and the right figures show the data of patients with IFX level $\geq$ by Wilcoxon signed-rank test.

528 Abbreviations: CDAI, clinical disease activity index; CRP, C-reactive protein; DAS28ESR, the 28 joint disease activity score incorporating erythrocyte sedimentation rate; HAQ-DI, physical disability by health assessment questionnaire-disability index; IFX, infliximab; SDAI, simplified disease activity index.

Fig. 5 Comparison of serum IFX levels between ADA positive group (ADA $(+))$ and negative group (ADA (-)). Each dot represents each patient's serum IFX level and the bars indicate the median. Differences between groups were assessed by Mann-Whitney 
U test.

537 Abbreviations: ADA, anti-drug antibody; IFX, infliximab.

538

539 Supplementary information

540 Additional file 1: Table S1. Number of responders and non-responders at maximum

541 effect and measurement point in High/Low-IFX groups.

542 The cut-off value was determined to be at serum IFX level $\geq 1.0 \mu \mathrm{g} / \mathrm{mL}$. Responders

543 had "good and moderate responses" and non-responders had "no responses" based on

544 the EULAR response criteria. Values were considered statistically significant at a $p$

545 value less than 0.05 , based on two-sided Fisher's exact test.

546 Abbreviations: EULAR, European League Against Rheumatism; IFX, infliximab. 
Table 1. Baseline demographics and clinical characteristics of the patients.

\begin{tabular}{lccc}
\hline \multicolumn{1}{c}{ Characteristics } & $\begin{array}{c}\text { IFX }<0.4 \mu \mathrm{g} / \mathrm{mL} \\
(\mathrm{n}=10)\end{array}$ & $\begin{array}{c}\text { IFX } \geq 0.4 \mu \mathrm{g} / \mathrm{mL} \\
(\mathrm{n}=31)\end{array}$ & $p$-value \\
\hline Age, mean (SD), (years) & $47.7(17.4)$ & $61.9(11.9)$ & $<0.01$ \\
Body weight, mean (SD), (kg) & $59.9(13.0)$ & $55.1(7.9)$ & 0.46 \\
Women, no. (\%) & $7(70.0)$ & $25(80.6)$ & 0.66 \\
Disease duration, mean (SD), (years) & $3.02(1.96)$ & $4.25(3.65)$ & 0.51 \\
Duration of IFX treatment, & $336(142-539)$ & $388(112-882)$ & 0.14 \\
$\quad$ & & & \\
median (Min-Max), (days) & $9.4(2.7)$ & $8.6(2.5)$ & 0.39 \\
(mg/weekly $\quad$ MTX dose, mean & (SD), & & \\
Oral glucocorticoid use, no. (\%) & $0(0.0)$ & $13(41.9)$ & $<0.01$ \\
csDMARDs use, no. (\%) & $2(20.0)$ & $8(25.8)$ & $>0.99$ \\
Tender joint count, mean (SD) & $2.6(3.6)$ & $5.1(5.6)$ & 0.06 \\
Swollen joint count, mean (SD) & $2.9(3.3)$ & $5.2(5.2)$ & 0.22 \\
CRP level, mean (SD), (mg/dL) & $1.6(3.3)$ & $2.2(3.0)$ & 0.49 \\
RF positive, no. (\%) & $9(90.0)$ & $21(67.7)$ & 0.24 \\
CDAI, mean (SD) & $12.7(8.9)$ & $19.9(13.4)$ & 0.03 \\
SDAI, mean (SD) & $14.3(12.0)$ & $22.1(15.2)$ & 0.03 \\
HAQ-DI, mean (SD) & $0.70(0.75)$ & $1.17(0.95)$ & 0.14 \\
DAS28-ESR, mean (SD) & $4.04(1.19)$ & $4.86(1.37)$ & 0.08 \\
\hline
\end{tabular}

550 The patients were divided into two groups; Low-IFX (serum IFX level $<0.4 \mu \mathrm{g} / \mathrm{mL}$ ) and

551 High-IFX (serum IFX level $\geq 0.4 \mu \mathrm{g} / \mathrm{mL}$ ). Demographics and clinical characteristics at

552 baseline are represented as means \pm standard deviation (SD) for continuous data and

553 numbers (percentages) for categorical data. Analysis of variance and Fisher's exact test

554 were used to compare the clinical characteristics among the different groups for

555 continuous variables and categorical variables, respectively. csDMARDs include actarit,

556 aurothiomalate, auranofin, bucillamine, iguratimod, leflunomide, mizoribine, 
557 salazosulfapyiridin, cyclosporine, and tacrolimus.

558 Abbreviations: CDAI, clinical disease activity index; csDMARDs, conventional 559 synthetic disease modifying anti-rheumatic drugs; CRP, C-reactive protein; DAS28-ESR, 560 the 28 joint disease activity score incorporating erythrocyte sedimentation rate; HAQ-DI, 561 physical disability by health assessment questionnaire-disability index; IFX, infliximab; 562 MTX, methotrexate; SDAI, simplified disease activity index; RF, rheumatoid factor. 563 
564 Table 2. Number (percentages) of responders and non-responders at maximum effect and 565 measurement point in each group.

\begin{tabular}{|c|c|c|c|}
\hline & Responders & Non-responders & $p$-value \\
\hline \multicolumn{4}{|l|}{$<$ maximum effect point $>$} \\
\hline $\mathrm{IFX}<0.4 \mu \mathrm{g} / \mathrm{mL}, \mathrm{n}(\%)$ & $9(90.0)$ & $1(10.0)$ & \multirow{2}{*}{0.43} \\
\hline IFX $\geq 0.4 \mu \mathrm{g} / \mathrm{mL}, \mathrm{n}(\%)$ & $30(96.8)$ & $1(3.2)$ & \\
\hline Total (\%) & $39(95.1)$ & $2(4.9)$ & \\
\hline \multicolumn{4}{|l|}{$<$ measurement point $>$} \\
\hline $\mathrm{IFX}<0.4 \mu \mathrm{g} / \mathrm{mL}, \mathrm{n}(\%)$ & $4(40.0)$ & $6(60.0)$ & \multirow{2}{*}{$<0.01$} \\
\hline IFX $\geq 0.4 \mu \mathrm{g} / \mathrm{mL}, \mathrm{n}(\%)$ & $30(96.8)$ & $1(3.2)$ & \\
\hline Total (\%) & $34(82.9)$ & $7(17.1)$ & \\
\hline \multicolumn{4}{|l|}{$<$ measurement point $>$} \\
\hline ADA-positive, n (\%) & $2(50.0)$ & $2(50.0)$ & \multirow{2}{*}{0.14} \\
\hline ADA-negative, n (\%) & $30(85.7)$ & $5(14.3)$ & \\
\hline Total $(\%)$ & $32(82.1)$ & $7(17.9)$ & \\
\hline
\end{tabular}

566 Responders had "good and moderate responses," and non-responders had "no responses"

567 based on the EULAR response criteria. ADAs in two patients could not be examined due

568 to sample shortage. Values were considered statistically significant at a $p$ value less than

$569 \quad 0.05$, based on two-sided Fisher's exact test.

570 Abbreviations: ADA, anti-drug antibody; EULAR, European League Against

571 Rheumatism; IFX, infliximab.

572 
Figure 1

RA patients using IFX between January 1, 2011 and December 31, 2018 (311 patients)

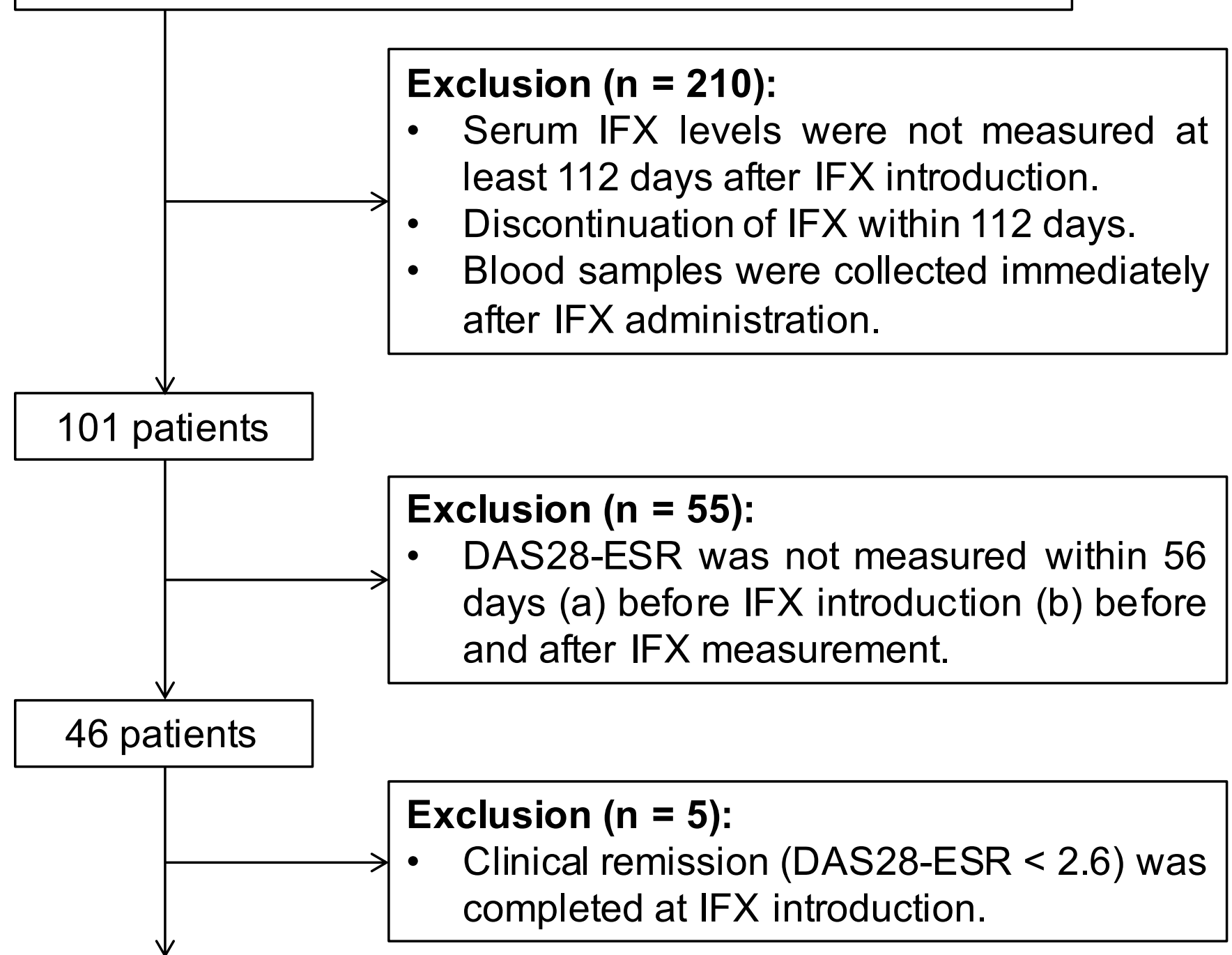

Study Cohort

41 patients 
Figure 2

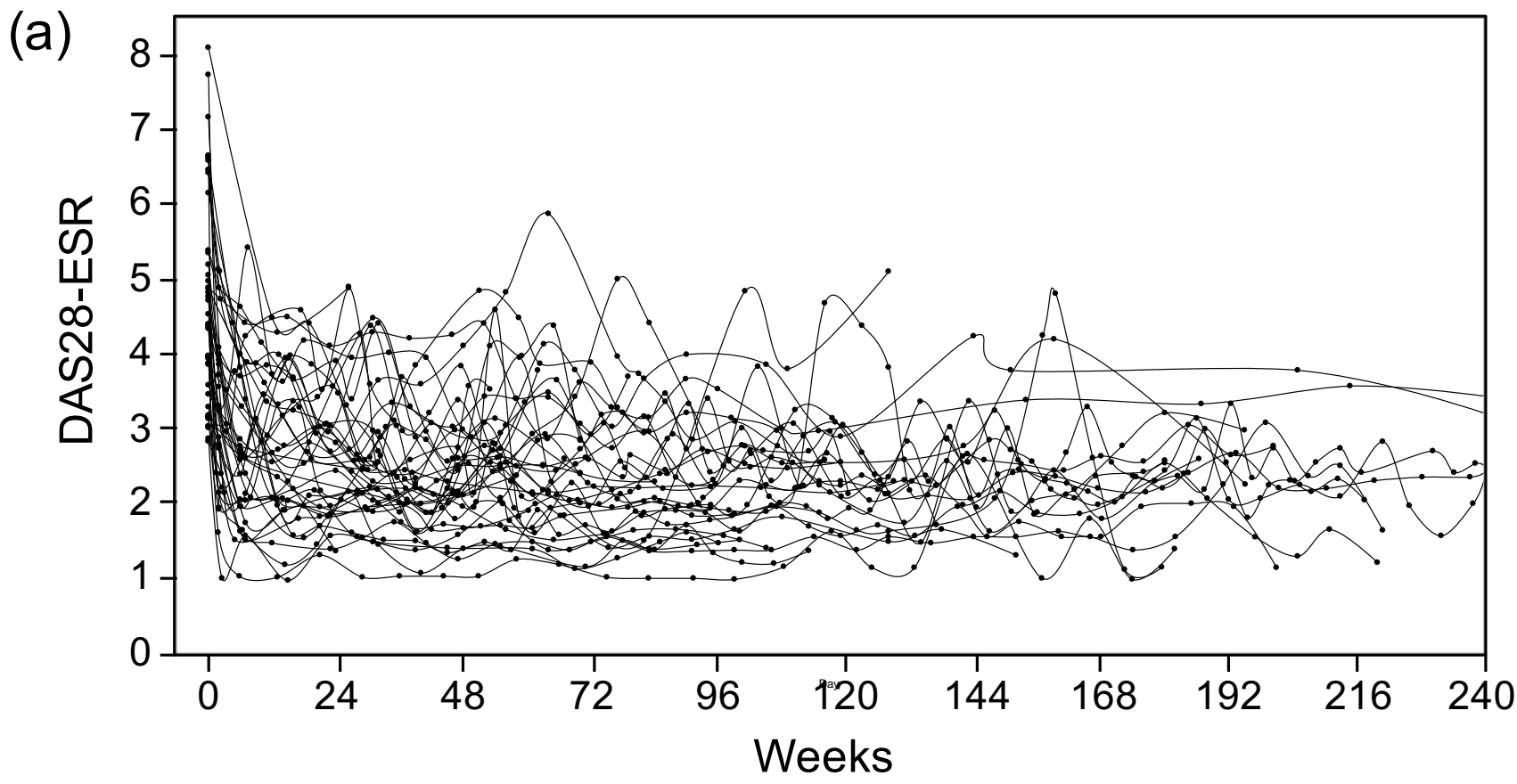

(b)

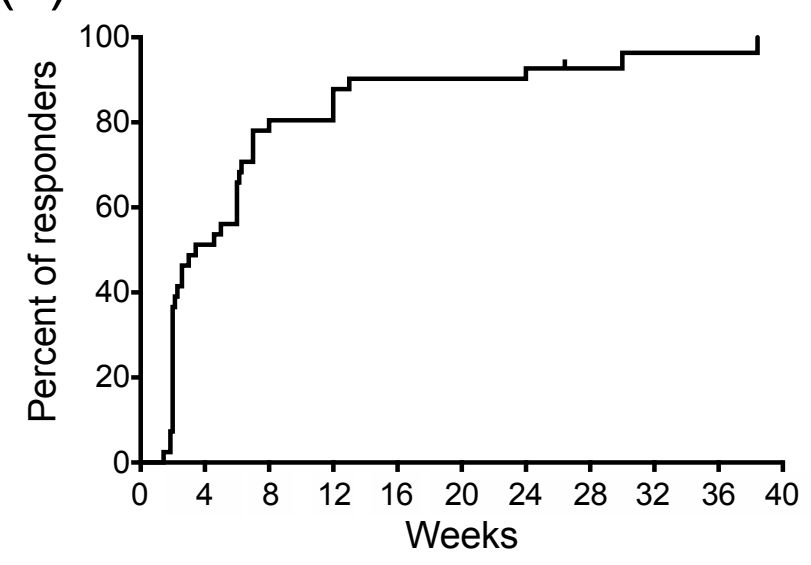

(c)

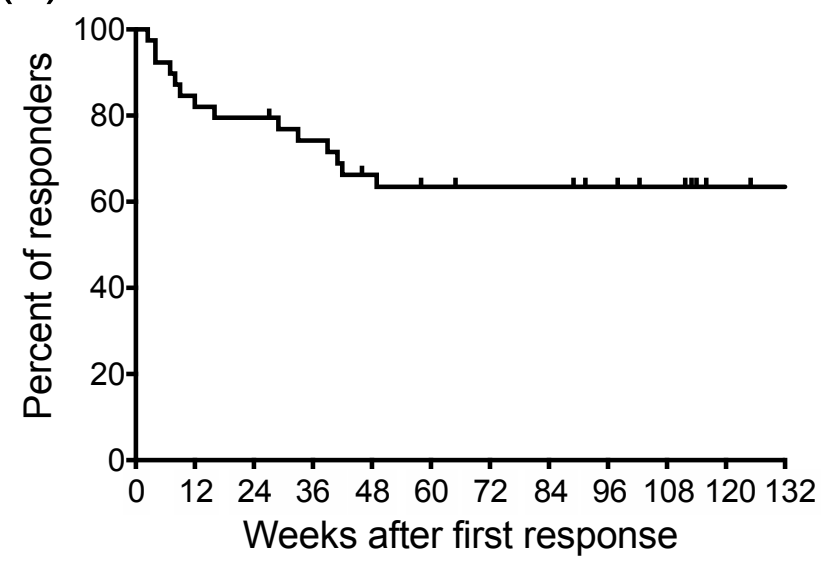


Figure 3

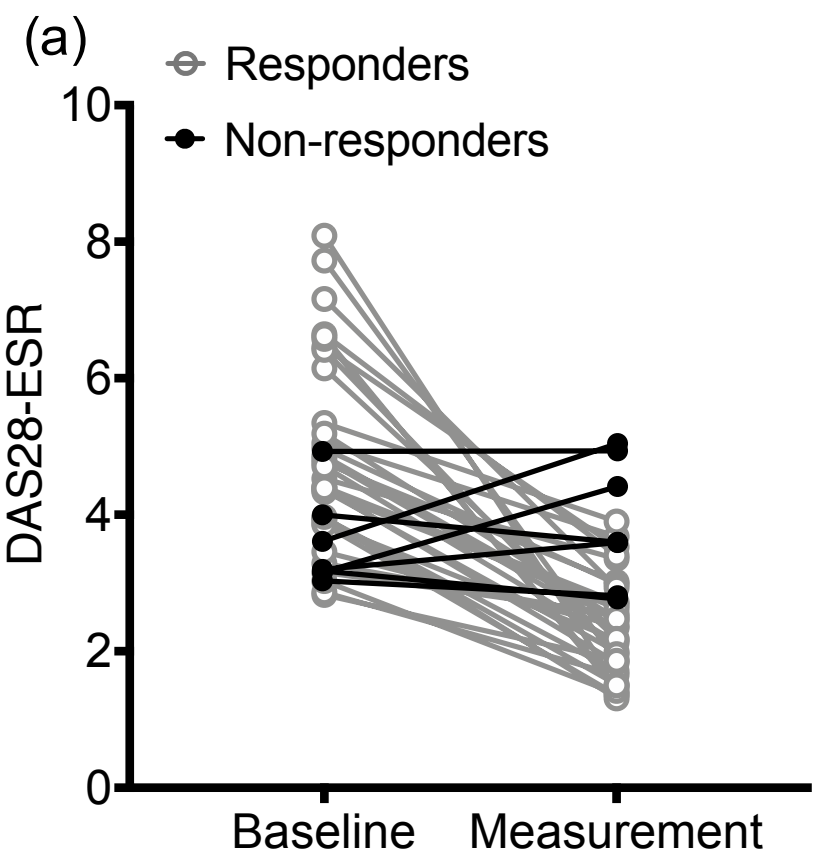

(b)

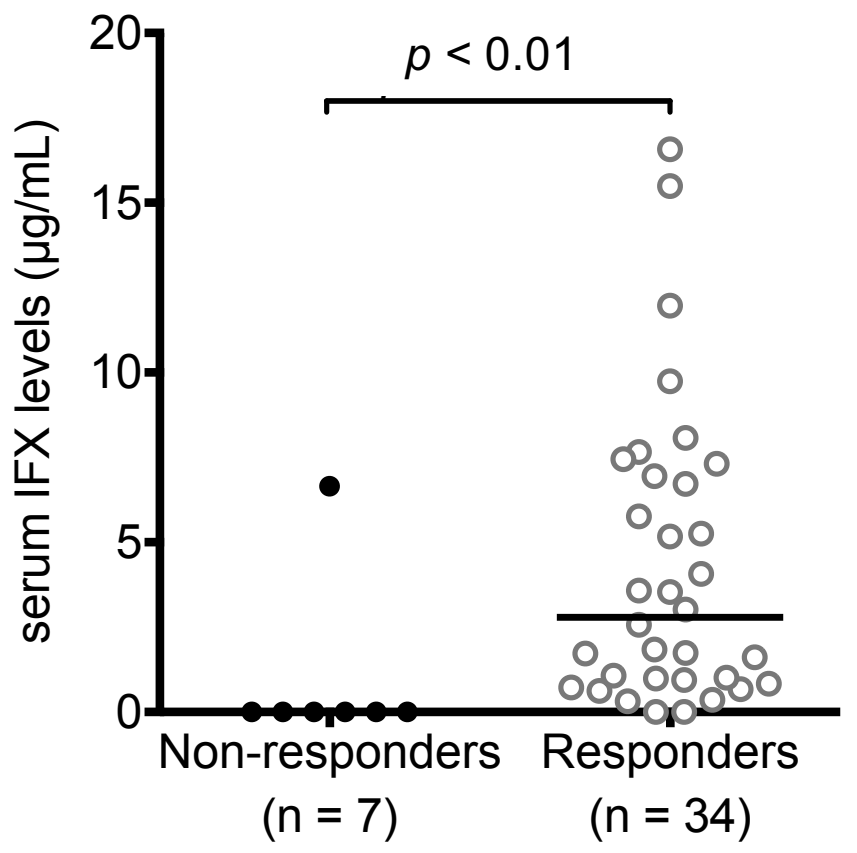

(c)

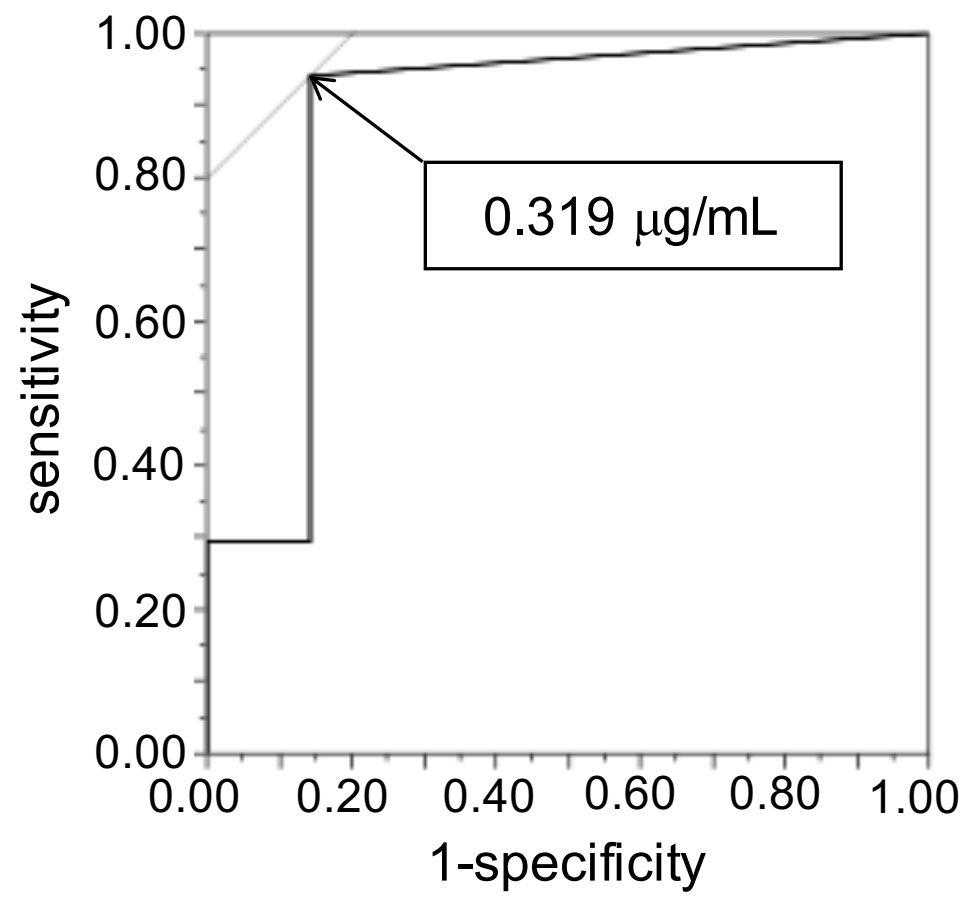


Figure 4

(a)

Low-IFX

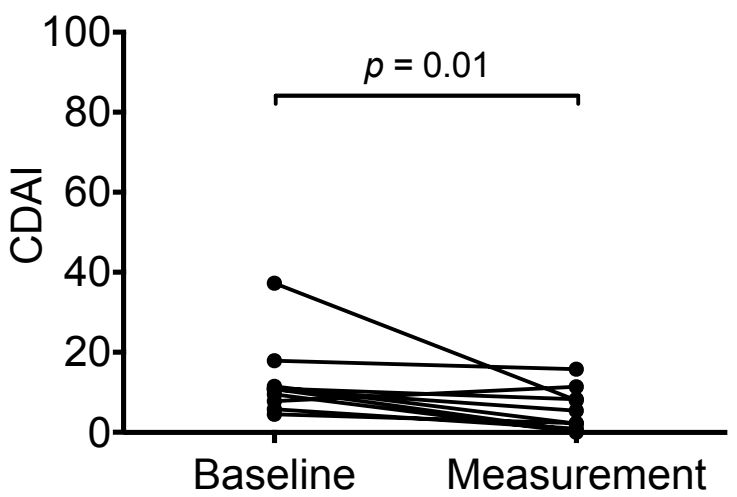

(b)

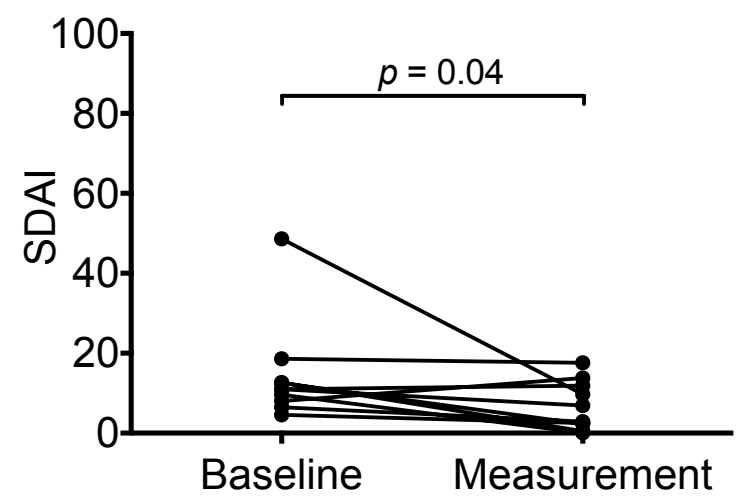

(c)

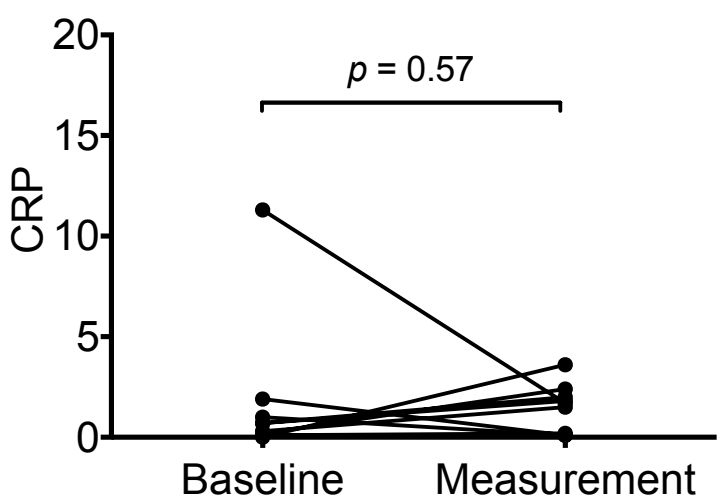

(d)

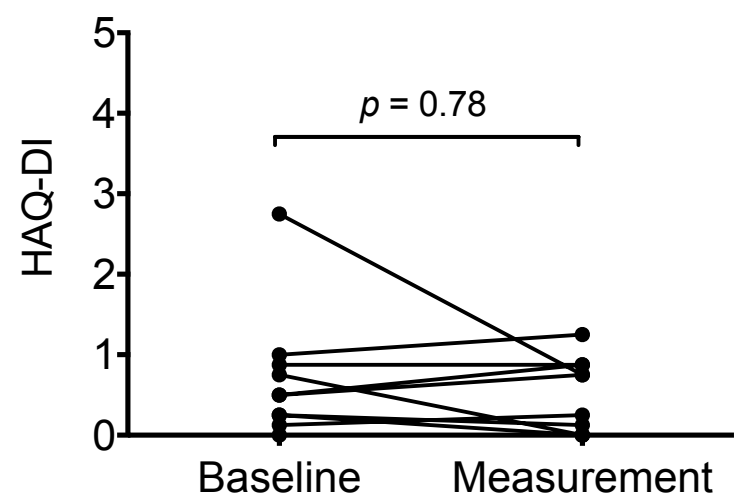

High-IFX

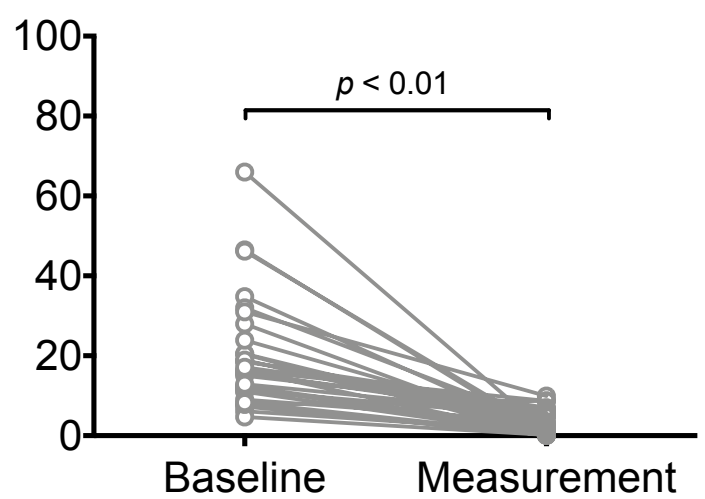

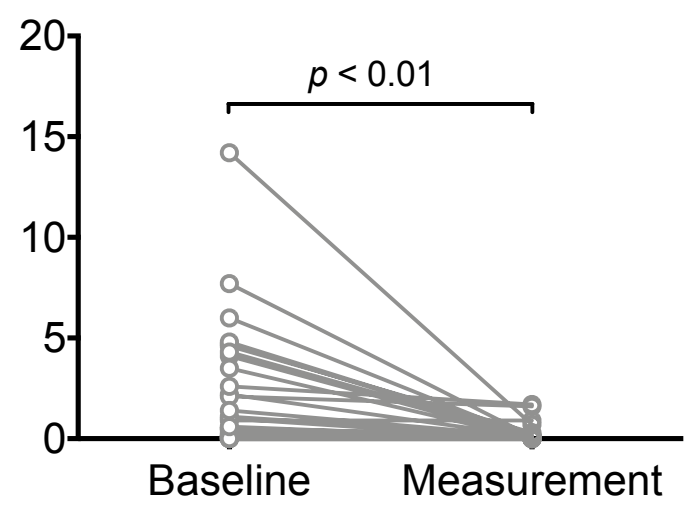

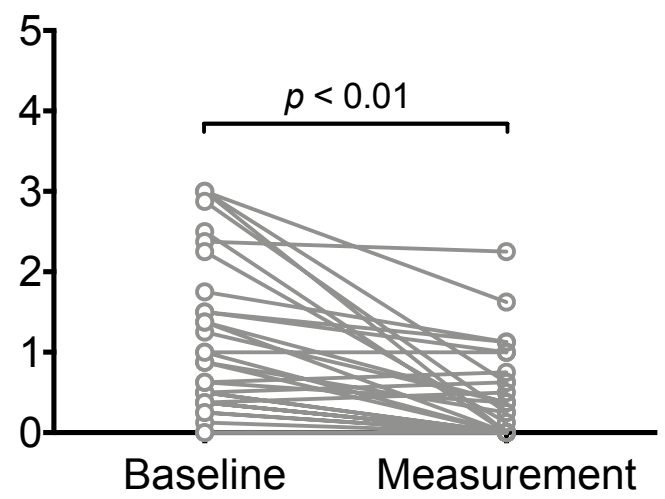


Figure 5

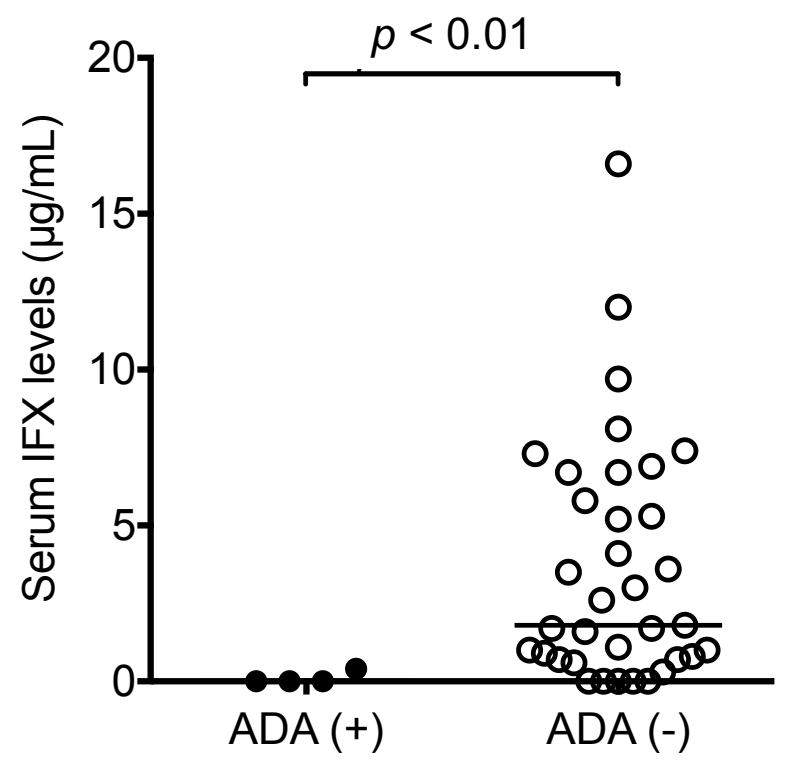




\section{Figures}

Figure 1

\begin{tabular}{l}
$\begin{array}{l}\text { RA patients using IFX between January 1, } 2011 \text { and } \\
\text { December 31, } 2018 \text { (311 patients) }\end{array}$ \\
\hline
\end{tabular}

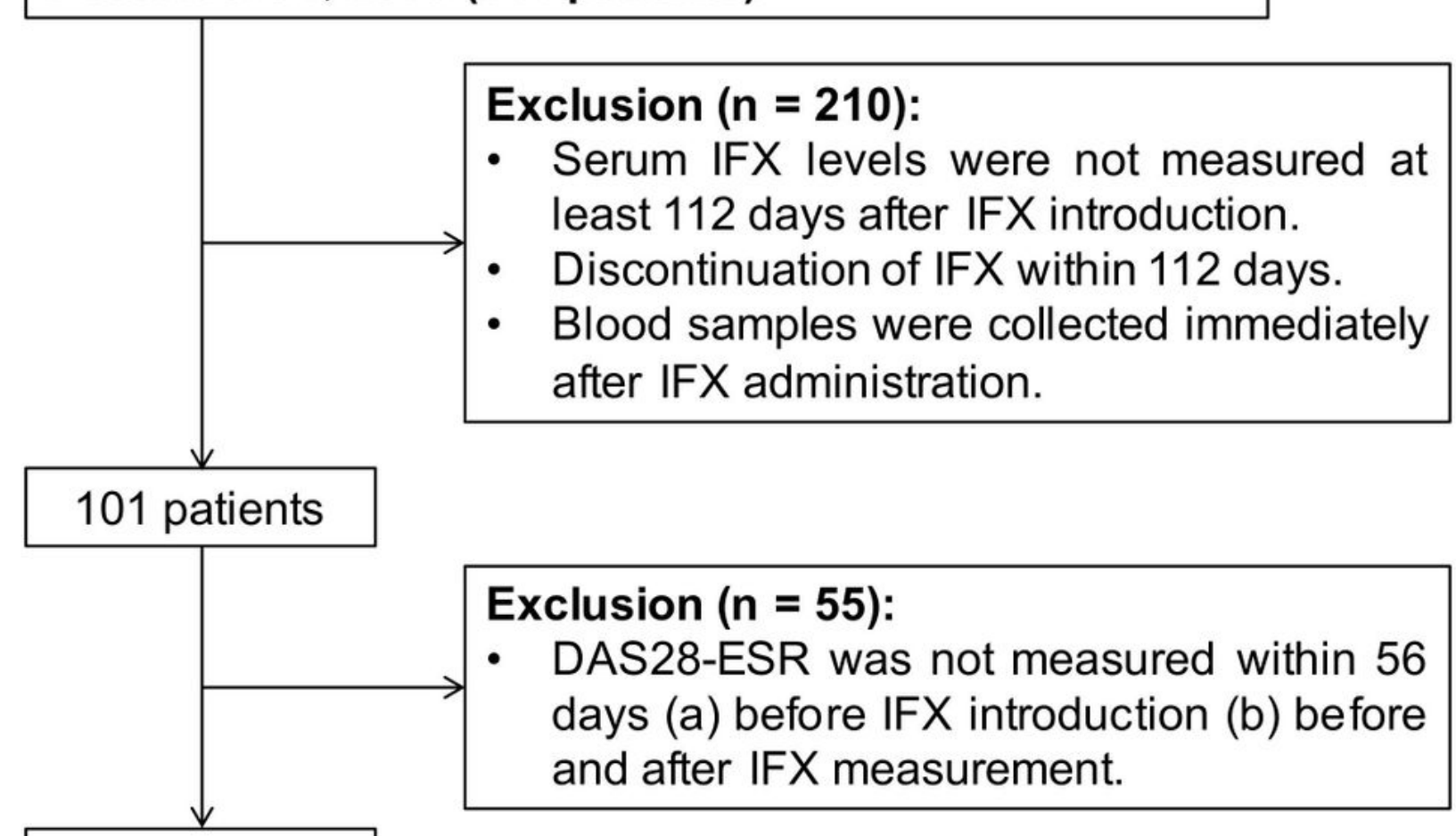

46 patients

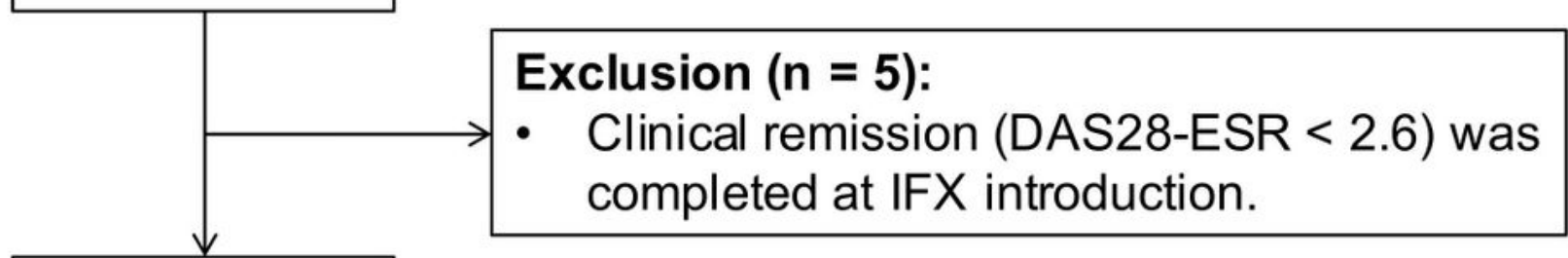

\section{Study Cohort}

41 patients

Figure 1

Please see the Manuscript PDF file for the complete figure caption 
Figure 2

(a)

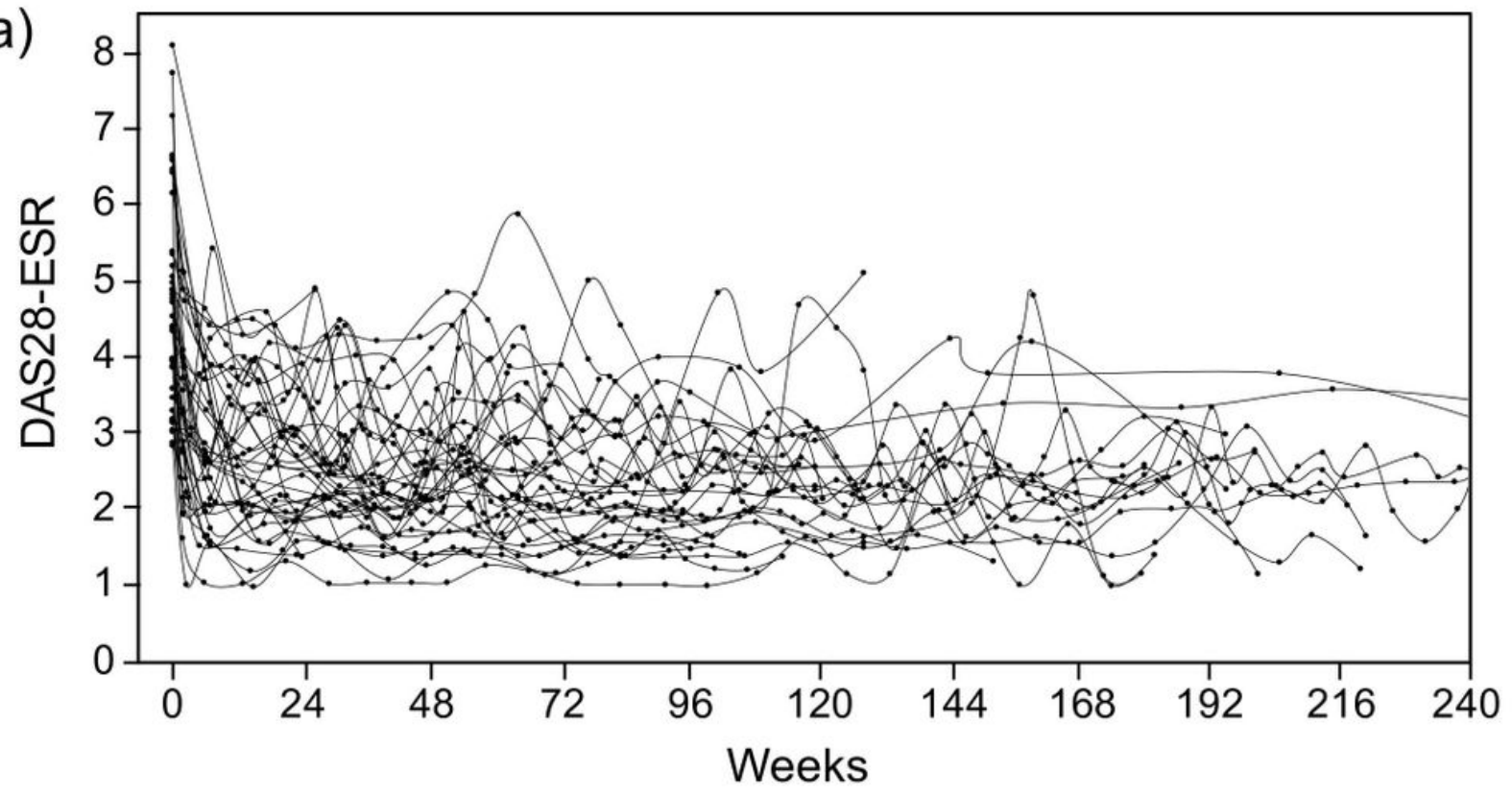

(b)

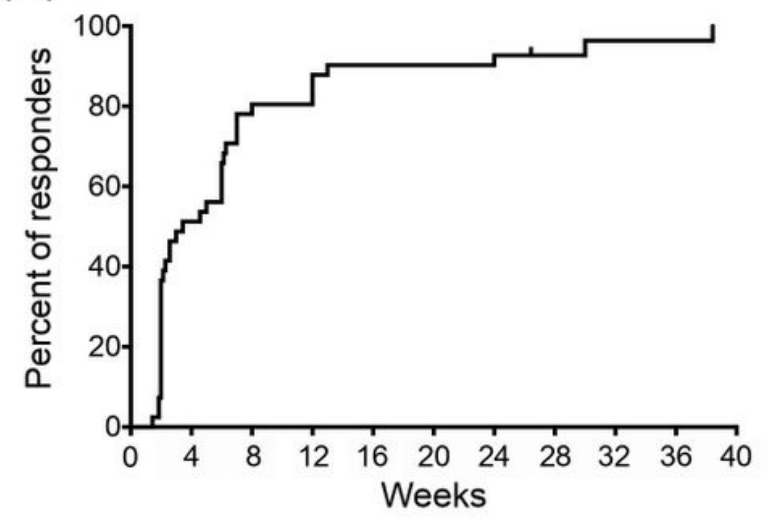

(c)

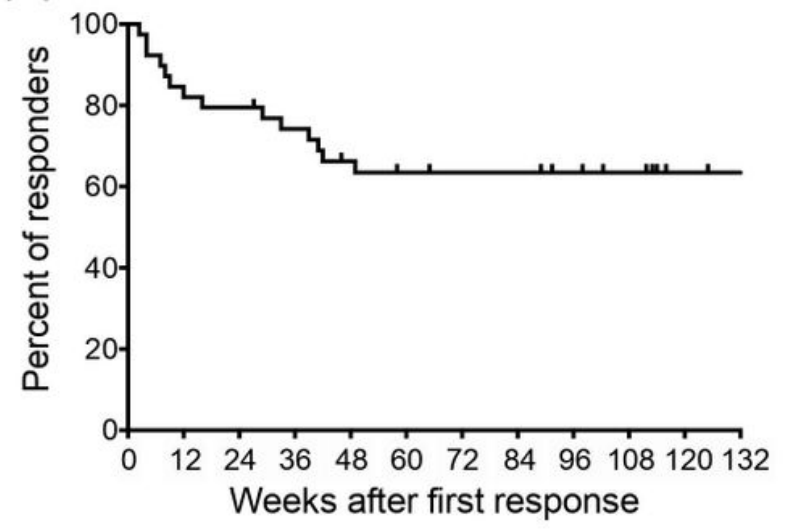

Figure 2

Please see the Manuscript PDF file for the complete figure caption 
Figure 3
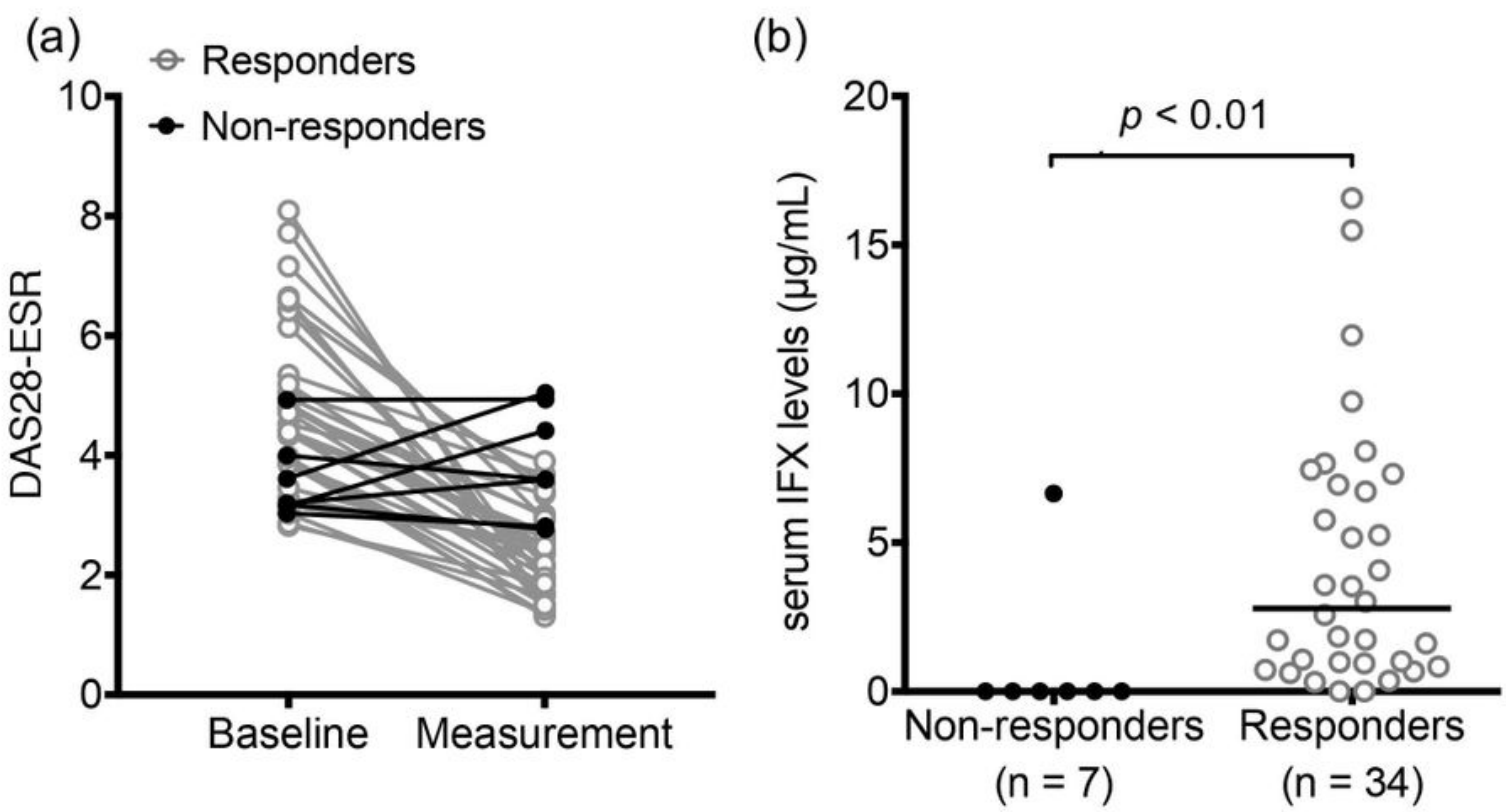

(c)

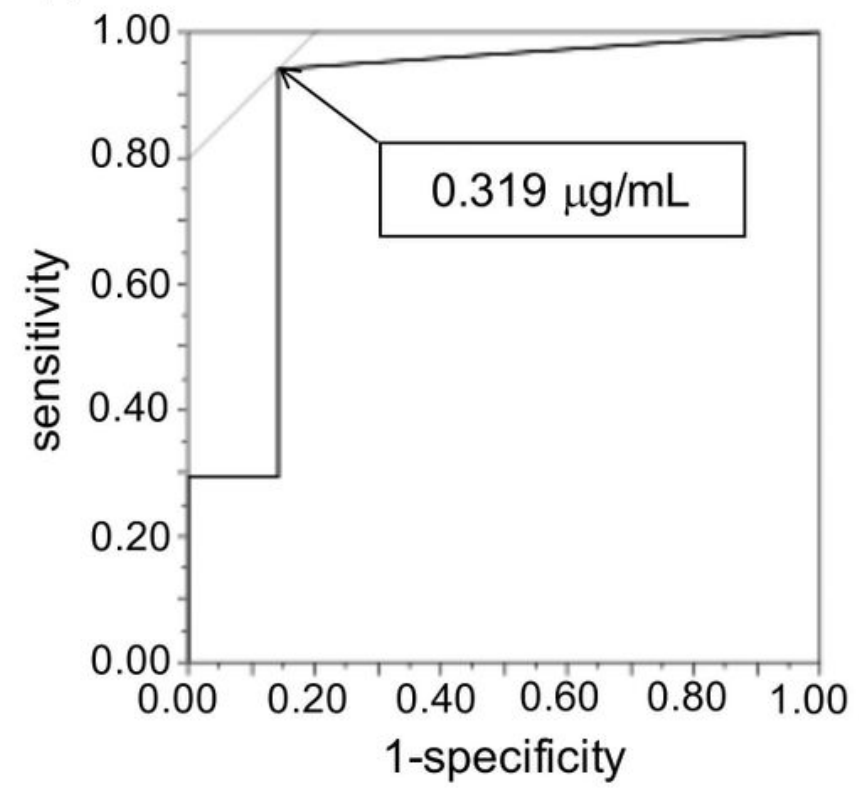

Figure 3

Please see the Manuscript PDF file for the complete figure caption 
Figure 4

(a)

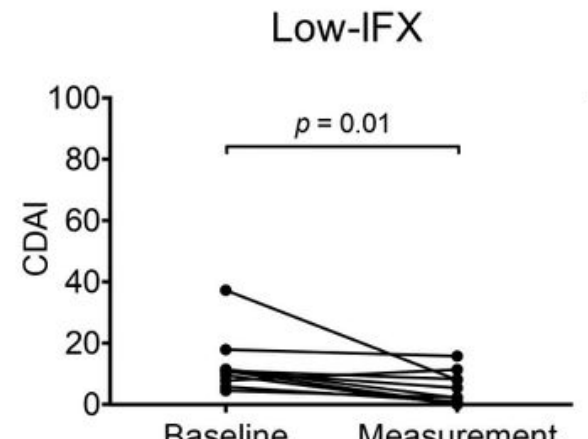

(b)

(c)
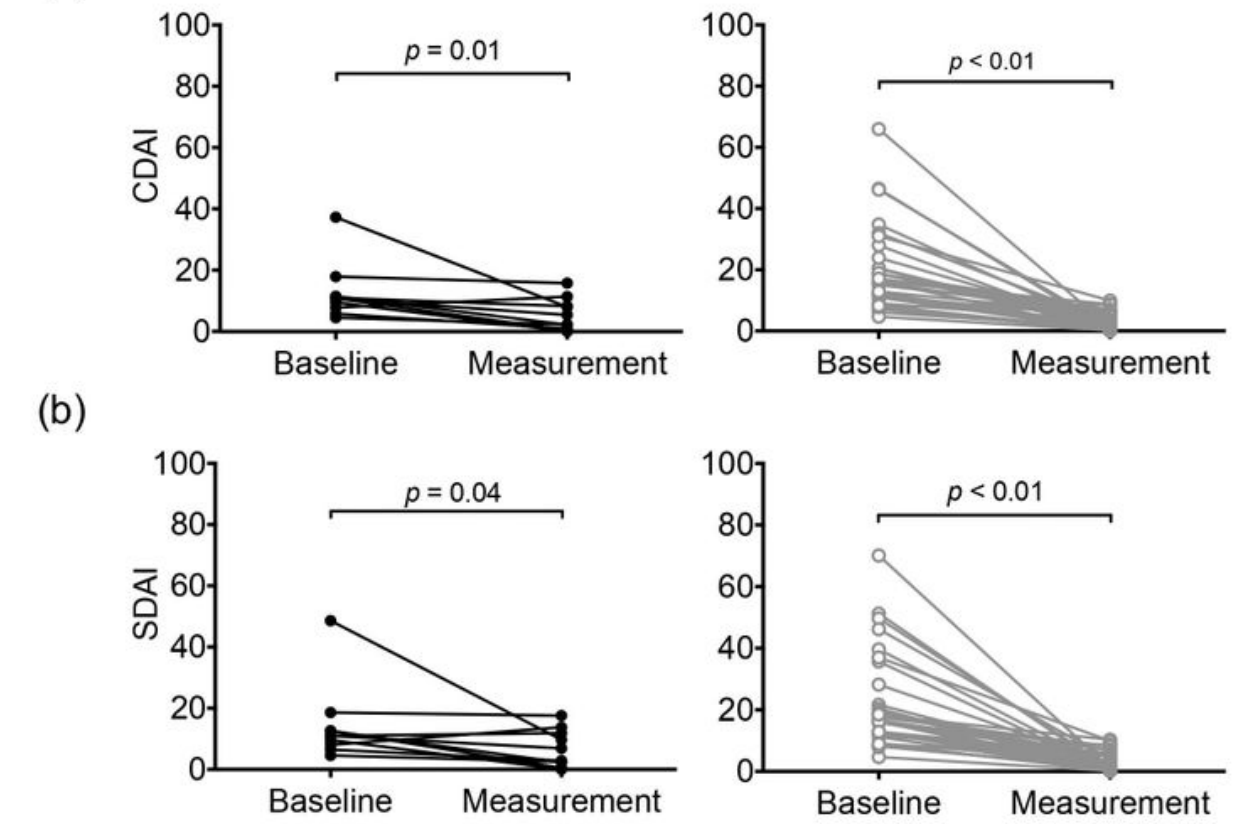

(d)
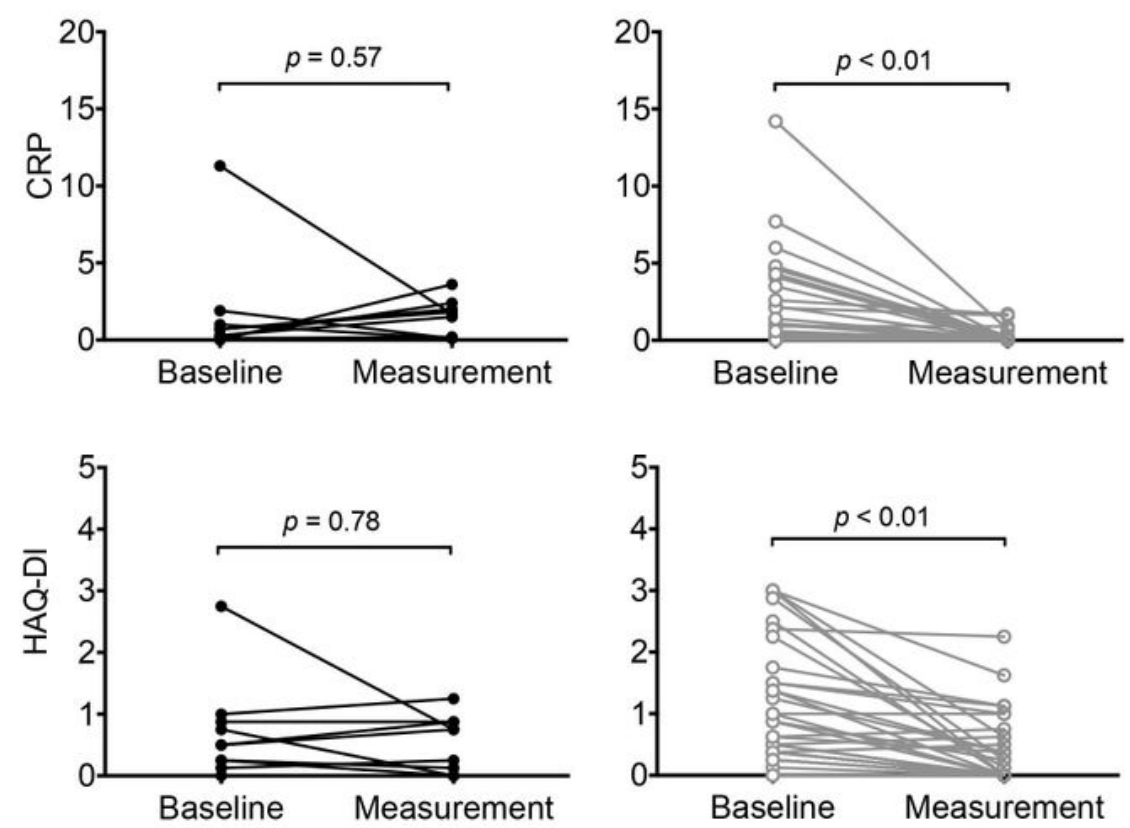

\section{Figure 4}

Please see the Manuscript PDF file for the complete figure caption 
Figure 5

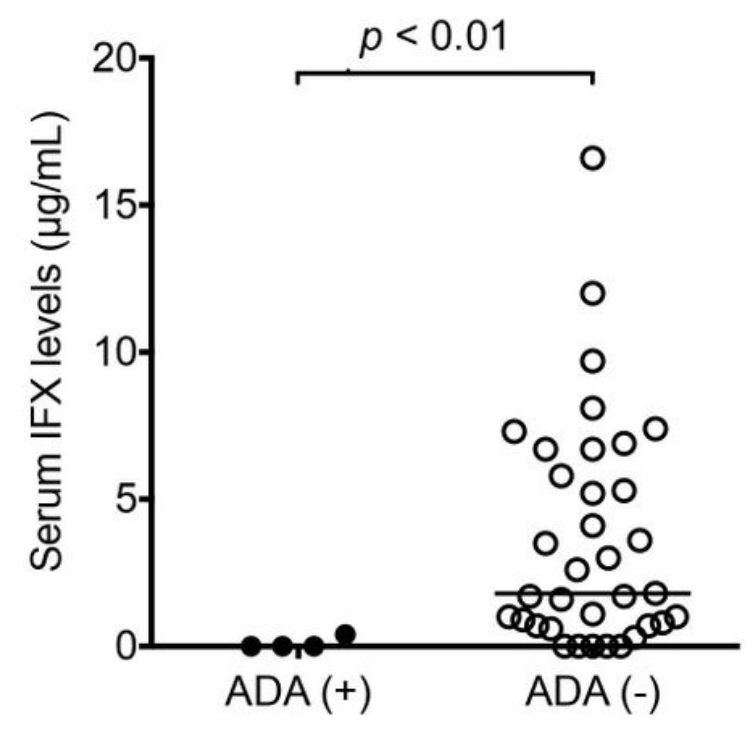

Figure 5

Please see the Manuscript PDF file for the complete figure caption

\section{Supplementary Files}

This is a list of supplementary files associated with this preprint. Click to download.

- Supplement.docx 